\title{
On the Crossing Number of Almost Planar Graphs
}

\author{
Petr Hliněný ${ }^{1, \star}$ and Gelasio Salazar ${ }^{2, \star \star}$ \\ ${ }^{1}$ Faculty of Informatics, Masaryk University \\ Botanická 68a, 60200 Brno, Czech Republic \\ hlineny@fi.muni.cz \\ ${ }^{2}$ Instituto de Física, Universidad Autónoma de San Luis Potosí \\ San Luis Potosí, Mexico, 78000 \\ gsalazar@ifisica.uaslp.mx
}

\begin{abstract}
Crossing minimization is one of the most challenging algorithmic problems in topological graph theory, with strong ties to graph drawing applications. Despite a long history of intensive research, no practical "good" algorithm for crossing minimization is known (that is hardly surprising, since the problem itself is NP-complete). Even more surprising is how little we know about a seemingly simple particular problem: to minimize the number of crossings in an almost planar graph, that is, a graph with an edge whose removal leaves a planar graph. This problem is in turn a building block in an "edge insertion" heuristic for crossing minimization. In this paper we prove a constant factor approximation algorithm for the crossing number of almost planar graphs with bounded degree. On the other hand, we demonstrate nontriviality of the crossing minimization problem on almost planar graphs by exhibiting several examples, among them new families of crossing critical graphs which are almost planar and projective.
\end{abstract}

Keywords: crossing number, crossing minimization, planarization, crossing-critical graphs.

2000 Math Subject Classification: 05C10, 05C62, 68R10.

\section{Introduction}

We assume that the reader is familiar with the standard notation of terminology of graph theory, and especially with topological graphs, see [11. In this paper we consider finite graphs, with multiple edges allowed.

The crossing number $\operatorname{cr}(G)$ of a graph $G$ is the minimum number of pairwise edge crossings in a drawing of $G$ in the plane (thus, a graph is planar if and only if its crossing number is 0$)$. A drawing of $G$ with $\operatorname{cr}(G)$ crossings is (crossing-) optimal. Crossing number problems were introduced by Turán, whose work in a brick factory during the Second World War led him to inquire about the

\footnotetext{
* Supported in part by the Institute of Theoretical Computer Science, project 1M0545.

** Supported by CONACYT Grant 45903.
} 
crossing number of the complete bipartite graphs $K_{m, n}$. It is remarkable that this long-standing particular question is still open. Not surprisingly, exact crossing numbers are in general very difficult to compute.

Nowadays, computing crossing numbers has important applications in VLSI design, and, naturally, in graph drawing. The algorithmic problem of crossing minimization is given as follows:

Input: A (multi)graph $G$ and an integer $k$.

Question: Is $\operatorname{cr}(G) \leq k$ ? (Possibly: if so, find an optimal drawing.)

The problem is in NP since one could guess the optimal drawing, replace the crossings in it with new (degree 4, subdividing) vertices, and verify planarity of the resulting graph. It has been proved by Garey and Johnson [4] that crossing minimization is NP-complete if $k$ is a part of the input. The same assertion has been proved true later by the first author [10] both for cubic graphs and for the minor-monotone version (cf. [1]) of crossing number. An important, stubborn open problem is to determine whether the crossing number of graphs with bounded tree-width can be computed in polynomial time.

On the positive side, a surprising result from Grohe states that the crossing number is an FPT parameter.

Theorem 1.1 (Grohe [6]). One can decide whether $\operatorname{cr}(G) \leq k$ for an $n$-vertex graph $G$ in time $O\left(f(k) \cdot n^{2}\right)$.

Grohe's algorithm is the only efficient algorithm (given fixed $k$ ) known so far for computing exact crossing numbers. Unfortunately, this algorithm is not usable in practice, not even for relatively small values of $k$, since $f$ is double exponential in $k$ and, moreover, the "hidden constants" are very large.

Regarding approximability results, the best result known to date is a (polynomial time) $\log ^{3} n$ approximation algorithm by Even, Guha and Schieber [3]. Constant factor approximation algorithms are known only for particular families of graphs, such as projective graphs with bounded degree [5].

Our paper brings two new main results to the theory of crossing numbers of almost planar graphs: First, Theorem 2.2 proves that a known heuristic for crossing minimization of an almost planar graph $G+e$ - take a suitable planar embedding of $G$ and insert $e$ to it - is a provably good approximation on graphs of bounded degrees. Second (on the negative side), Theorem 3.4 brings new rich families of $k$-crossing-critical graphs which are both almost planar and projective, that is, as close to planarity as one can reasonably imagine.

\section{Approximating the Crossing Number of Bounded-Degree Almost Planar Graphs}

Currently, it seems that the best known general-purpose practical heuristic approach to crossing minimization on a graph $G$ is the following: First, delete from $G$ some (small set of) edges $F$, so that $G^{\prime}=G-F$ is planar. Then, take an 
edge $f \in F$ and a suitable planar embedding of $G^{\prime}$, and find a way of inserting $f$ back to the drawing of $G^{\prime}$ with the smallest number of crossings (using a shortest-path algorithm on the topological dual of $G^{\prime}$ ). After that, create a new graph $G^{\prime \prime}$ from $G^{\prime}+f$ by replacing the crossings with new vertices, and iterate the process with $G^{\prime \prime}$ and $F \backslash\{f\}$.

This heuristic algorithm outlines the following interesting subproblem on almost-planar graphs (recall that a graph is almost planar if it has an edge whose removal leaves a planar graph), hereafter called the one-edge crossing minimization:

Input: A planar graph $G$ and two nonadjacent vertices $u, v$ of $G$.

Problem: Find an optimal drawing of $G+u v$ (i.e. $G$ plus the edge $u v$ ), that is, a drawing with the minimum number of crossings.

Although we firmly believe that computing the crossing number of an almost planar graph cannot be an NP-hard problem, all our efforts to get a polynomial time algorithm failed (even for graphs with bounded degrees). Thus we moved on to investigate whether such a crossing number can be approximated by a polynomial time algorithm. Our aim in this section is to show the existence of such an approximation algorithm, for graphs with bounded degrees.

Attempts to solve the one-edge crossing minimization problem, in turn, have brought a closely related subproblem with the same input, hereafter called the one-edge bridging minimization for distinction. This modification asks for a planar embedding of $G$ such that inserting the edge $u v$ to it yields the minimum possible number of crossings. Let $\operatorname{br}(G, u v)$ denote the minimum number of crossings in the bridging minimization problem.

The one-edge bridging minimization problem has been completely solved, giving a linear-time optimal algorithm for it, by Gutwenger, Mutzel and Weiskircher 78]. As they observe, that algorithm does not necessarily yield an optimal solution to the crossing minimization problem, as the counterexample at the end of [8] (thereby attributed to G. Farr) shows. This is summarized in the following statement.

Proposition 2.1. For each $k>2$ there is a planar graph $G$, and vertices $u, v$, such that $\operatorname{cr}(G+u v)=2$, but $\operatorname{br}(G, u v)=k$.

It is interesting to mention that this was asked as an open question in the earlier conference version 7 . We have independently found a somewhat simpler counterexample, which is (for $k=5$ ) illustrated in Fig. 1 .

We note that both Farr's construction and our example make essential use of vertices of large degree (of order at least $2 k$ ). It is thus natural to ask whether large degree vertices are an essential part of any such examples. Our following result settles this question - large degree vertices are indeed unavoidable.

Theorem 2.2. Suppose that $G$ is a planar graph with maximum degree $\Delta$, and let $u, v$ be nonadjacent vertices of $G$. Then the one-edge bridging minimization problem on $G$ and uv has an optimal solution with $\operatorname{br}(G, u v) \leq \Delta \cdot \operatorname{cr}(G+u v)$. 

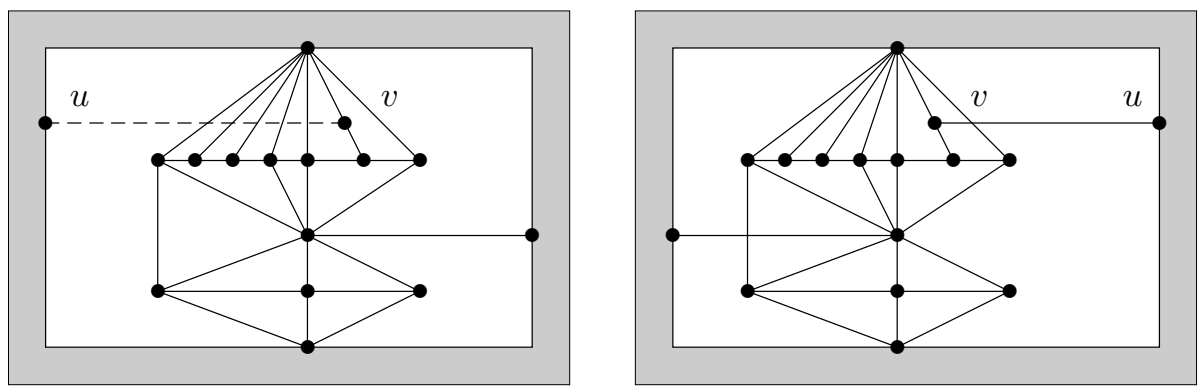

Fig. 1. A counterexample showing that a solution to one-edge bridging minimization (left, dashed edge $u v$ ) can be arbitrarily far from the crossing number (right). The shadow area stands for a sufficiently dense planar part.

We prove the theorem later in this section, using further Lemma 2.4. Our statement has the following nice consequence:

Corollary 2.3. There is a polynomial time approximation algorithm for computing the crossing number of an almost planar graph with bounded degrees.

Proof. Let $G$ be an almost planar graph with maximum degree $\Delta$. Apply any efficient planarity algorithm to find an edge $e$ of $G$ such that $G-e$ is planar. Then apply the linear time algorithm for one-edge bridging minimization from 8 to obtain a planar embedding of $G-e$ such that inserting $e$ into it yields the minimum possible number of crossings, say $k$. Obviously $\operatorname{cr}(G) \leq k$, and it follows from Theorem 2.2 that $k / \Delta \leq \operatorname{cr}(G)$.

If $\Psi, \Psi^{\prime}$ are embeddings of the same graph $G$, then $\Psi^{\prime}$ is a mirror embedding of $\Psi$ if there is an orientation reversing homeomorphism taking $\Psi$ to $\Psi^{\prime}$.

Suppose that $G$ has a 2 -cut $\{x, y\}$, and let $G_{1}, G_{2}$ be subgraphs of $G$ such that $G$ is the sum of $G_{1}$ and $G_{2}$ along $x$ and $y$ (that is, $G_{1}$ and $G_{2}$ are edgedisjoint and share only $x$ and $y$, and $G=G_{1} \cup G_{2}$ ). A (Whitney) flipping at $x, y$ is a re-embedding of $G$ such that the embedding of $G_{2}$ is unchanged and the embedding of $G_{1}$ is a mirror of the original embedding. We say that $G_{1}, G_{2}$ are the sides of the flipping. Any two (combinatorial) embeddings of a 2-connected planar graph can be transformed to each other by a sequence of flippings. We call a flipping prime if one of $G_{1}$ or $G_{2}$ has no cut-vertex separating $x$ from $y$. Obviously, every flipping can be decomposed into prime flippings.

Lemma 2.4. Suppose $H$ is a connected plane graph (i.e. actually embedded in the plane), and e, $f$ are two edges not belonging to $H$ but connecting vertices of $H$, such that $H+f$ is a planar graph. If e can be drawn in $H$ with $\ell$ crossings, then there is a planar embedding of $H+f$ in which $e$ can be drawn with at most $\ell+2 \cdot\lfloor\Delta(H) / 2\rfloor$ crossings.

It is worth noting that the statement may be false if $H$ is disconnected and $e, f$ join vertices from two distinct components. 


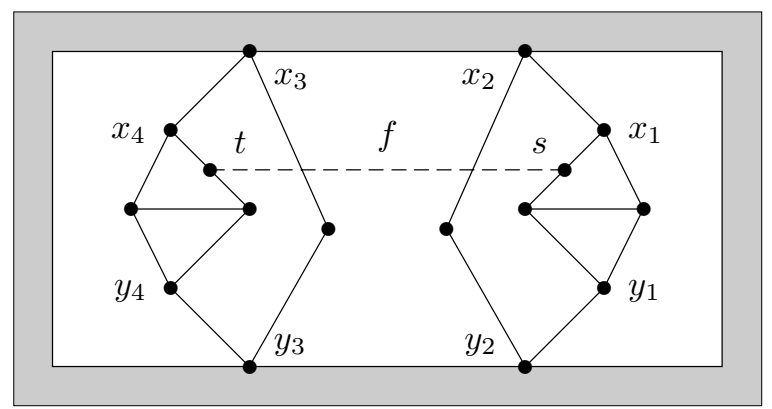

Fig. 2. An example of a planar graph in which four flippings are necessary before the edge $f$ (with endpoints $s$ and $t$ ) can be embedded in without crossings

Proof. First we assume that $H$ is 2-connected. Consider a sequence of flippings which turn $H$ into an embedding $H^{\prime}$ such that $H^{\prime}+f$ is plane (although we talk about a sequence, the order of the flippings actually does not matter to us). As noted above, we may assume the flippings under consideration are prime. Note that each of the sides of any relevant flipping contains one endpoint of $f$. We naturally order by inclusion the set of all the sides (of all the relevant flippings) containing an end $s$ of $f$, say as $S_{1} \subset S_{2} \subset \ldots \subset S_{m}$ (see also Fig. 2). Let $x_{i}, y_{i}$ be the cut-vertices at which the flipping involving $S_{i}$ occurs.

Observe further that it is enough to consider those flippings that have exactly one endpoint of $e$ on each side as well. (If there is no such flipping, then $e$ can be drawn with the same number of crossings in plane $H^{\prime}+f$ as in $H$ itself.) Let $i, 1 \leq i \leq m$, be the smallest index such that $S_{i}$ contains an endpoint of $e$. Thus, no endpoint of $e$ is in $S_{i-1}$ if $i>1$, and exactly one endpoint $u$ of $e$ is in $S_{i}$. After we apply the flippings of $S_{1}, \ldots, S_{i-1}$, the vertex $s$ has to appear on the unbounded face of $S_{i}$ due to planarity of $H+f$ (note that those flippings do not affect the drawing or number of crossings on the edge $e$ ). We look at the "half-edge" $e_{0} \subset e$ from the end $u$ till reaching the unbounded face of $S_{i}$ (for this moment we regard the edge $e$ as a topological object in the drawing $H+e$ ): We extend the curve $e_{0}$ to $e_{1}$ so that its loose end appears in a close neighbourhood of the vertex $s$ on the unbounded face of $S_{i}$, which takes only at most $\left\lfloor d_{H}\left(x_{i}\right) / 2\right\rfloor$ or $\left\lfloor d_{H}\left(y_{i}\right) / 2\right\rfloor$ additional crossings on $e_{1}$ when passing by either vertex $x_{i}$ or $y_{i}$, respectively. (See Fig. 3.)

A symmetric argument can be simultaneously used to argue that the other "half-edge" $e_{0}^{\prime} \subset e$ from the end $v$ of the edge $e=u v$ can be redrawn as $e_{1}^{\prime}$ such that its loose end appears in a close neighbourhood of the endpoint $t$ of the edge $f$. Then we apply the remaining flippings and embed the edge $f$ into $H^{\prime}$. Finally, we join the loose ends of $e_{1}$ and $e_{1}^{\prime}$ together along the (uncrossed) edge $f$ in $H^{\prime}+f$, producing no additional crossing with $f$ since we have had above a choice of redrawing of $e_{1}$ either by $x_{i}$ or $y_{i}$. In this way we get a drawing of $e$ inside the plane graph $H^{\prime}+f$ with at most $2 \cdot\lfloor\Delta(H) / 2\rfloor$ additional crossings. 


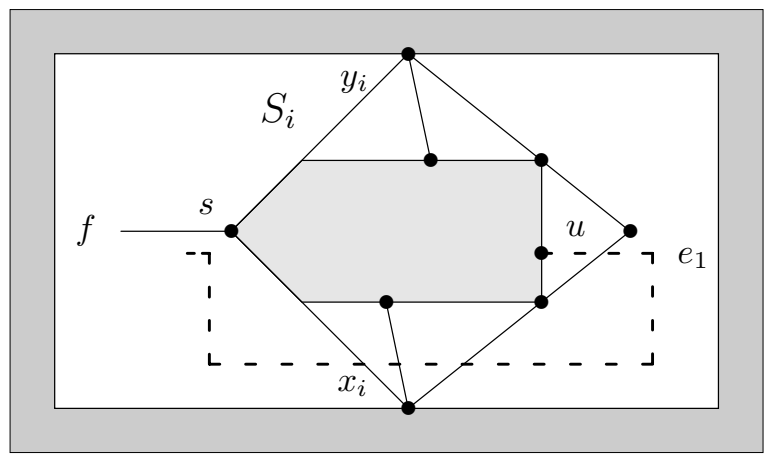

Fig. 3. An illustration of the proof of Lemma 2.4

At last we have to consider connected $H$ which is not 2-connected. Although all deep arguments have been used already above, some boring technical details are still necessary, and we only sketch them here for simplicity. Imagine $H$ decomposed into blocks. If the decomposition contains a leaf block not incident with $e, f$, then we may simply delete it. If the decomposition contains a leaf block incident with $f$ but not with $e$, then we may contract this block into one vertex without changing our problem. If the decomposition contains a leaf block incident with $e$ but not with $f$, then similarly we may contract this block into one vertex and just make a note of the number of crossings $e$ had with this block.

After processing the above reductions, we either arrive at a 2-connected graph, or we get a graph with precisely two leaf blocks $B_{1}, B_{2}$, both incident with $e$ and $f$. Then we adapt the above "half-edge" argument to this situation: We redraw the half-curve of $e$ from $B_{1}$ so that its loose end appears in a close neighbourhood of the cut-vertex $b_{1}$ of the block $B_{1}$, in the unbounded face. Symmetrically, we redraw the half-curve of $e$ from $B_{2}$ so that its loose end appears in a close neighbourhood of the cut-vertex $b_{2}$ of $B_{2}$. Finally we join the two halves of $e$ together in the unbounded face. This again costs at most $2 \cdot\lfloor\Delta(H) / 2\rfloor$ additional crossings.

Having the previous lemma at hand, it is easy to finish the proof of Theorem 2.2. We actually prove a stronger statement (indeed, to see that Theorem 2.2 follows from Lemma 2.5, it suffices to note that $m \leq \operatorname{cr}(G+u v)-\ell)$.

Lemma 2.5. Suppose that $G$ is a planar graph with maximum degree $\Delta$, and let $u, v$ be nonadjacent vertices of $G$. If there is a crossing-optimal drawing of $G+u v$ such that the edge uv has $\ell$ crossings, and removing $m$ edges from this drawing of $G$ makes it plane, then $\operatorname{br}(G, u v) \leq \ell+2\lfloor\Delta / 2\rfloor m$.

Proof. Let $F$ be a set of edges of $G$ such that $G^{\prime}=G-F$ is plane and $|F|=m$. Obviously, for a crossing-optimal drawing $G+u v$ and minimal $F$, the graph $G^{\prime}$ is connected. Let $F=\left\{f_{1}, \ldots, f_{m}\right\}$. For $i=1,2, \ldots, m$, we apply Lemma 2.4 
for $H=G^{\prime}+f_{1} \ldots+f_{i-1}$ and $f=f_{i}, e=u v$, and continue with the resulting embedding of $H+f$ in the next iteration until $i=m$.

Remark. One may also consider an analogous $k$-edge crossing minimization problem for fixed $k>1$. Although the related $k$-edge bridging minimization problem [2, Problem 29] seems to have no efficient solution yet; if that is eventually found, then similar arguments could be used to prove it provides a good approximation for crossing minimization in the case of bounded degrees.

\section{Projective Almost Planar Graphs and Crossing-Critical Graphs}

Almost planar graphs are interesting both theoretically and practically. Although it is trivial to construct almost planar graphs with arbitrarily large crossing numbers (a large grid plus an edge joining vertices far apart from each other), a strong objection to such examples is that that the graph thus obtained is, in a way, not as close to being planar as it could be. Indeed, such a graph would have Euler genus 0 (since it is toroidal), thus being one step further away from planar than another large, interesting family, namely the collection of graphs with Euler genus 1. Recall that a graph has Euler genus 1 if and only if it is projective, that is, embeddable in the projective plane (equivalently, embeddable in the Möbius band).

Our aim in this section is to make several remarks on the richness of projective almost planar graphs. In our own view, this is the first step in a systematic program to understanding almost planar graphs: as we observed above, among almost planar graphs, projective graphs are arguably the simplest ones. We first observe that almost planar projective graphs can have arbitrary crossing number, and yet be strongly connected and have a small number of vertices (especially when compared to the number of vertices in the "grid example").

Proposition 3.1. For every $k$, there is a simple 4-connected graph on $2 k+4$ vertices which is almost planar and projective, and whose crossing number is $k$.

Proof. Let $D_{m}$ denote the double-wheel with the rim circuit of length $m$, and let $F_{k}$ be the graph obtained from $D_{2 k+2}$ by joining one pair of opposite vertices on

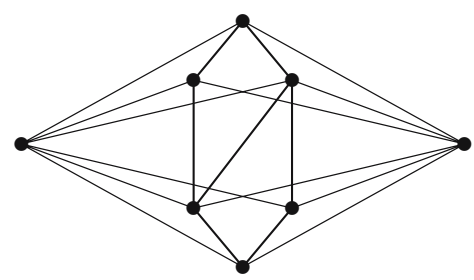

Fig. 4. The graph $F_{2}$ in the proof of Lemma 3.1 - a double-wheel of length 6 with an extra chord 
the rim circuit with edge $f$. This graph is projective since one may use a "Möbius twist" applied to $f$ and $k$ consecutive spokes of one of the wheels (between the ends of $f$ ) to embed it.

By an easy induction on $k$ we show $\operatorname{cr}\left(F_{k}\right)=k$. For $k=1, F_{k}$ is a subdivision of $K_{5}$, and so this is true. Again an argument with nonplanarity of $K_{5}$-subdivisions in $F_{k}$ shows that in any drawing of $F_{k}$ there has to be a length-2 path between the centers of the wheels that is crossed. Hence we remove the edges of this path and an opposite path (saving 1 crossing), and apply the inductive assumption for the resulting subdivision of $F_{k-1}$.

If we admit the possibility of multiple edges, analogous ideas can be used to find even smaller graphs with similar properties.

Proposition 3.2. For every $\ell$, there is a graph with $4 \ell+6$ edges which is both almost planar and projective, and whose crossing number is $\ell$.

Proof. This graph $\left(C_{\ell+2} \oplus K_{2}^{\ell}\right)$ is obtained from the disjoint union of $C_{\ell+2}$ and $\ell$ parallel edges $K_{2}^{\ell}$ by adding all edges between them. (Notice that this graph is actually $\ell$-crossing-critical, but since it is not simple, this does not make a "good" crossing-critical family.) The proof is very similar to that of Proposition 3.1

If, moreover, we bound the maximal degree, we observe the following.

Proposition 3.3. Suppose a graph $G$ is almost planar and projective, and the maximum degree of $G$ is bounded. Then the crossing number of $G$ is bounded as well, and so it can be computed in time $O\left(n^{2}\right)$.

Proof. According to [12, any projective embedding of an almost planar graph $G$ (more generally of an apex graph) has "face-width at most two". That precisely means the embedding admits a closed noncontractible curve intersecting $G$ just in two vertices $u, v$. Cutting the projective plane along this curve, one gets a planar embedding with two copies of $u$ and two of $v$ on the unbounded face. It is clearly possible to pairwise identify those two copies of $u$ and those of $v$, with an introduction of $\left\lfloor d_{G}(u) / 2\right\rfloor \cdot\left\lfloor d_{G}(v) / 2\right\rfloor$ crossings. Hence $\operatorname{cr}(G)$ is bounded if the degrees are bounded, and we may use Theorem 1.1 .

We finally show that almost planar projective graphs are rich enough to provide nontrivial examples of crossing-critical graphs. Recall that a graph $G$ is $k$-crossing-critical if $\operatorname{cr}(G) \geq k$ but $\operatorname{cr}(G-e)<k$ for all edges $e \in E(G)$. Crossing-critical graphs are of great importance in the theory of crossing numbers, for them giving an insight into the structural properties that force large crossing numbers. (Notice that, in any graph of crossing number $k$, by successive deleting of suitable edges we always find a $k$-crossing-critical subgraph. Hence if a graph class contains a rich subclass of crossing-critical graphs, then the crossing-minimization problem is likely not trivial on that class.)

A rich family of almost planar simple 3 -connected $k$-crossing critical graphs for every $k \geq 3$ has been constructed by the first author in 9]. Looking at our 
second restriction, the family of [9] is not projective, but we have succeeded in modifying that construction to obtain the following.

Theorem 3.4. For every $k \geq 3$, there is an infinite family of simple 3 -connected $k$-crossing critical graphs which are almost planar and projective.

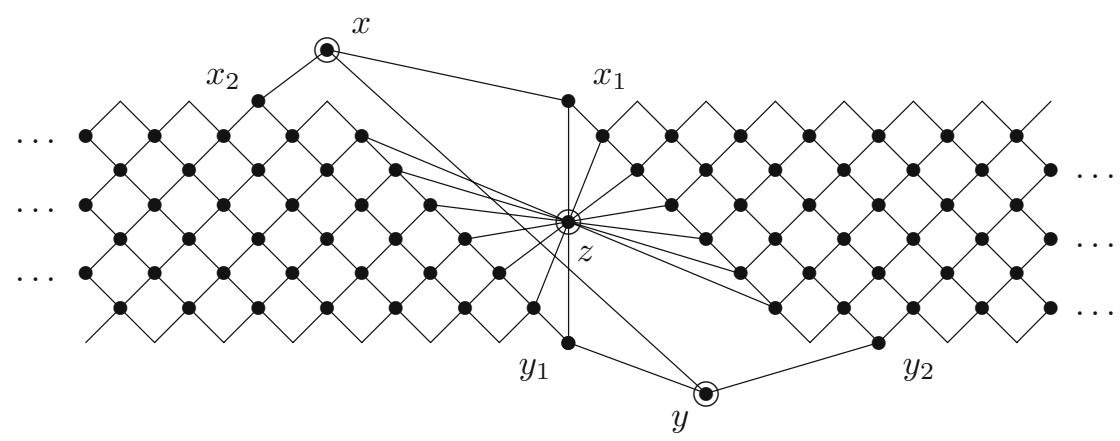

Fig. 5. An illustration of a $k$-belt graph, $k=6$ here. The left- and right-hand sides close together as on a cylinder.

The construction of graphs in Theorem 3.4 is illustrated in Fig. [5. and we call them $k$-belt graphs. Formally for $k \geq 1$, a graph $G$ with distinguished three vertices $x, y, z$ is a $k$-belt graph if the following are satisfied:

- $G$ is simple of minimum degree 3 if $k>2$.

- $B=G-\{x, y\}$ is formed as an edge-disjoint union of $k$ cycles sharing the vertex $z$. These cycles are denoted by $C_{1}, C_{2}, \ldots, C_{k}$ from top to bottom referring the picture in Fig. [6 left.

- The neighbourhood of $z$ in $B$ looks exactly as depicted in Fig. 5 , The neighbourhoods of other vertices of $B$ that are not adjacent to $z$ have all "squaregrid" structure (with a small exception at $C_{1}$ and $C_{k}$ ).

- There are two special vertices $x_{1}, x_{2} \in V\left(C_{1}\right) \backslash V\left(C_{2}\right)$ adjacent both to $x$ in $G$, such that $x_{1}$ is adjacent to $z$ while the distance of $x_{2}$ to $z$ is at least 3 . Another two special vertices $y_{1}, y_{2} \in V\left(C_{k}\right) \backslash V\left(C_{k-1}\right)$ adjacent both to $y$ in $G$ are defined symmetrically around $z$. Moreover, in the subgraph $B-z$, it holds that $x_{1}$ is farther to $x_{2}$ than to $y_{2}$, and $y_{1}$ is farther to $y_{2}$ than to $x_{2}$. (To better understand this condition, check that it implies the length of $C_{1}$ is at least $k+6$.)

- $x y$ is an edge in $G$. (Notice that $G-x y$ is a planar graph.)

We start with some straightforward statements about $G$ (see again Fig. (5)).

Lemma 3.5. If $G$ is a $k$-belt graph, $k \geq 1$, then

a) $G$ is almost planar, projective, and simple 3 -connected if $k \geq 3$,

b) $\operatorname{cr}(G) \geq k$ but $\operatorname{cr}(G-e)<k$ for all $e \in E(G)$. 

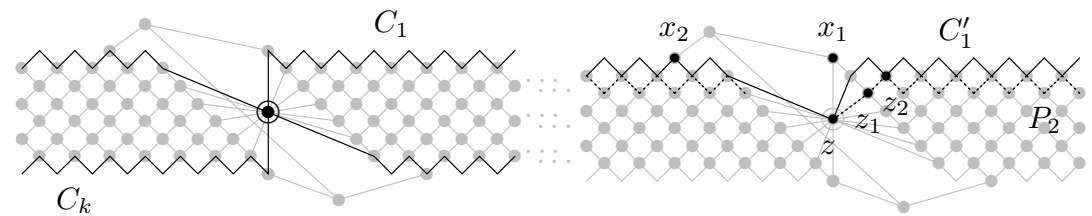

Fig. 6. Notation in $k$-belt graphs: (left) the cycles $C_{1}$ and $C_{k}$; (right) the cycle $C_{1}^{\prime}$, vertices $z, z_{1}, z_{2}$, and dotted path $P_{2}$

To complete the proof of Theorem 3.4, it now remains to argue why a $k$-belt graph cannot be drawn with less than $k$ crossings. For further arguments, we denote by $C_{1}^{\prime}$ the cycle in $B$ obtained from $C_{1}$ by deleting $x_{1}$ and using instead the edge from $z$ to the other neighbour of $x_{1}$ (Fig. 6 right). The following is easy to see from the definition of a $k$-belt graph.

Lemma 3.6. Suppose $G$ is a $k$-belt graph, $k \geq 3$, and there is an optimal drawing of $G$ with $\ell$ crossings such that the cycle $C_{1}^{\prime}$ is crossed. Let $G^{\prime}$ be the subgraph formed by the edges $\left(E(G) \backslash E\left(C_{1}^{\prime}\right)\right) \cup\left\{x_{2} x_{2}^{\prime}\right\}$, where $x_{2}^{\prime}$ is one of the neighbours of $x_{2}$ in $C_{1}^{\prime}$ such that $x_{2} x_{2}^{\prime}$ is not the only crossed edge of $C_{1}^{\prime}$. Then $G^{\prime}$ is a subdivision of a $(k-1)$-belt graph, and $\operatorname{cr}\left(G^{\prime}\right) \leq \ell-1$.

In the opposite situation, i.e. when $C_{1}^{\prime}$ is not crossed, we argue similarly. Let us denote by $z_{1}$ the neighbour of $z$ on $C_{2}$ and by $z_{2}$ the subsequent vertex on $C_{2}$. See in Fig. 6 right. The path starting with $z, z_{1}, z_{2}$ and continuing then along the vertices of $C_{2}$ up to the last one before returning to $z$ is denoted by $P_{2}$.

Lemma 3.7. Suppose $G$ is a $k$-belt graph, $k \geq 4$, and there is an optimal drawing of $G$ with $\ell$ crossings such that $C_{1}^{\prime}$ is not crossed. Then there are at least two distinct crossings on the path $P_{2}$. Consequently, there is a drawing $G^{\prime} \subseteq G$ which is a subdivision of a $(k-2)$-belt, and $\operatorname{cr}\left(G^{\prime}\right) \leq \ell-2$.

Proof. Since $G-V\left(C_{1}^{\prime}\right)$ is a connected subgraph, all its vertices have to be drawn in one of the faces bounded by the drawing of $C_{1}^{\prime}$. Hence by Jordan's curve theorem, the length-2 path $z z_{1} z_{2}$ has to be crossed since it separates the common neighbour of $z$ and $x_{1}$ from the rest of the drawing. The same can be said about the length- 2 path on $P_{2}$ which connects the two vertices adjacent to $x_{2}$. Those are the desired two distinct crossings on $P_{2}$.

Lemma 3.8. Suppose $G$ is a $k$-belt graph, $k=1$ or $k \geq 3$. Then $\operatorname{cr}(G) \geq k$.

Proof. We proceed by an induction on $k$, similarly as we have done in [9]. Notice that a 1-belt graph is actually a subdivision of $K_{3,3}$, and hence the base of induction holds. The main complication comes from the fact that the inductive statement is false for $k=2$, and we have to carefully avoid this in our arguments.

First consider $k>4$, and the statement is true for all $i<k, i \neq 2$. Take an optimal drawing of $G$ with $\ell$ crossings. Then, depending on whether the cycle $C_{1}^{\prime}$ is crossed, apply Lemma 3.6 or 3.7 straightforwardly: For $a=1$ or $a=2$, it 
is $k-a \leq \operatorname{cr}\left(G^{\prime}\right) \leq \ell-a$, and hence $k \leq \ell$. Next consider $k=4$. If Lemma 3.6 applies to $G$, induction proceeds in the same way. So assume $C_{1}^{\prime}$ is not crossed now, and by Lemma 3.7 there are two crossings on the path $P_{2}$. The same arguments can be applied to symmetrically defined cycle $C_{4}^{\prime}$ and path $P_{3}$ in $G$, and we find additional two crossings on $P_{3}$ (which is disjoint from $P_{2}$ ); altogether $4=k$ crossings.

It remains to prove the statement for $k=3$. This is straightforward but slightly too long for this restricted conference paper, and so we only sketch the arguments. If $C_{1}^{\prime}$ is not crossed, then we may still consistently apply Lemma 3.7 and induction. Otherwise, considering symmetry, both cycles $C_{1}^{\prime}$ and (symmetric) $C_{3}^{\prime}$ are crossed. (It may happen that $C_{1}^{\prime}$ crosses $C_{3}^{\prime}$.) Let a cycle $C_{2}^{\prime}$ be obtained from $C_{2}$ by replacing the two edges from $C_{2} \cap\left(C_{1}^{\prime} \cup C_{3}^{\prime}\right)$ with the path through $y_{1}, z, x_{1}$. If the cycle $C_{2}^{\prime}$ is crossed as well, then we apply an inductive step with removing (most of) edges of $C_{1}^{\prime}$ and $C_{2}^{\prime}$. Finally, if $C_{2}^{\prime}$ is not crossed at all, then one can show that $C_{1}^{\prime}$ and $C_{3}^{\prime}$ carry at least three distinct crossings, since in such case the vertices $x, y, x_{2}, y_{2}$ have to be drawn in the same face bounded by the drawing of $C_{2}^{\prime}$.

We remark that the above $k$-belt construction could be generalized in a similar way as the construction in 9] was. We however skip such a generalization here to avoid the boring technical details of it.

\section{Conclusions}

In this paper we mainly wanted to attract attention and research to the seemingly simple, but still quite deep and unexplored problem of crossing minimization on almost planar graphs. As our first step, we have shown two new results - one on the positive side (Theorem 2.2 and Corollary 2.3), and the other one somehow negative (Theorem 3.4), indicating richness and nontriviality of the problem we study.

Questions to the effect of whether crossing minimization remains hard even if we focus our attention on restricted families of graphs can be interpreted as partial efforts to answer an admittedly vague, but nonetheless appealing and still wide-open, question: How much "nonplanarity" must one admit into a family of graphs $\mathcal{G}$ in order to guarantee that computing the crossing number of a graph in $\mathcal{G}$ is hard?

We put forward three questions, in order of apparent difficulty.

Question 4.1. Is there a polynomial-time algorithm to approximate the crossing number of an almost planar graph?

Question 4.2. Is there a polynomial-time algorithm to compute the crossing number of an almost planar graph?

A simplified version of this last question would consider graphs with bounded degree, as we have done in the present paper. 
For the next question, recall than a graph $G$ is apex if it has a vertex $v$ such that $G-v$ is planar.

Question 4.3. Is it an NP-hard problem to compute the crossing number of an apex graph?

We conjecture that all these questions have affirmative answers. We lastly remark that Question 4.3 has been asked also by Mohar [private communication].

\section{References}

1. D. Bokal, G. Fijavz, and B. Mohar, The minor crossing number, SIAM Journal on Discrete Mathematics, to appear.

2. F. Brandenburg, D. Eppstein, M.T. Goodrich, S. Kobourov, G. Liotta and P. Mutzel, Selected Open Problems in Graph Drawing, In: Graph Drawing, GD 2003, Lecture Notes in Computer Science 2912, Springer Verlag 2004, 515-539.

3. G. Even, S. Guha, B. Schieber, Improved Approximations of Crossings in Graph Drawings and VLSI Layout Areas, SIAM J. Comput. 32(1), 231-252 (2002).

4. M.R. Garey, D.S. Johnson, Crossing number is NP-complete, SIAM J. Algebraic Discrete Methods 4 (1983), 312-316.

5. I. Gitler, J. Leaños, and G. Salazar, The crossing number of a projective graph is quadratic in the face-width. Manuscript (2006).

6. M. Grohe, Computing Crossing Numbers in Quadratic Time, J. Comput. Syst. Sci. 68 (2004), 285-302.

7. C. Gutwenger, P. Mutzel, R. Weiskircher, Inserting an edge into a planar graph, Symposium on Discrete Algorithms SODA 2001, SIAM (2001), 246-255.

8. C. Gutwenger, P. Mutzel, R. Weiskircher, Inserting an edge into a planar graph, Algorithmica 41 (2005), 289-308.

9. P. Hliněný, Crossing-critical graphs and path-width, In: Graph Drawing, 9th Symposium GD 2001, Vienna Austria, September 2001; Lecture Notes in Computer Science 2265, Springer Verlag 2002, 102-114.

10. P. Hliněný, Crossing number is hard for cubic graphs, J. of Combinatorial Theory ser. B 96 (2006), 455-471.

11. B. Mohar, C. Thomassen, Graphs on surfaces, Johns Hopkins Studies in the Mathematical Sciences, Johns Hopkins University Press (2001), Baltimore MD, USA.

12. N. Robertson, P.D. Seymour, R. Thomas, A survey of linkless embeddings, In: Graph Structure Theory (N. Robertson, P. Seymour, eds.), Contemporary Mathematics, American Mathematical Society, 1993, 125-136. 